\title{
Frameless robot-assisted stereoelectroencephalography in children: technical aspects and comparison with Talairach frame technique
}

\author{
Taylor J. Abel, MD, ${ }^{1,2}$ René Varela Osorio, MD, ${ }^{1}$ Ricardo Amorim-Leite, MD, ${ }^{3}$ \\ Francois Mathieu, MD, ${ }^{2}$ Philippe Kahane, MD, PhD, ${ }^{3}$ Lorella Minotti, MD, ${ }^{3}$ \\ Dominique Hoffmann, MD, ${ }^{1}$ and Stephan Chabardes, MD' \\ ${ }^{1}$ Clinique de Neurochirurgie, and ${ }^{3}$ Clinique Neurologique, Centre Hospitalier Universitaire Grenoble-Alpes, Grenoble, France; \\ and 2 Division of Neurosurgery, The Hospital for Sick Children, Toronto, Ontario, Canada
}

\begin{abstract}
OBJECTIVE Robot-assisted stereoelectroencephalography (SEEG) is gaining popularity as a technique for localization of the epileptogenic zone (EZ) in children with pharmacoresistant epilepsy. Here, the authors describe their frameless robot-assisted SEEG technique and report preliminary outcomes and relative complications in children as compared to results with the Talairach frame-based SEEG technique.
\end{abstract}

METHODS The authors retrospectively analyzed the results of 19 robot-assisted SEEG electrode implantations in 17 consecutive children (age < 17 years) with pharmacoresistant epilepsy, and compared these results to 19 preceding SEEG electrode implantations in 18 children who underwent the traditional Talairach frame-based SEEG electrode implantation. The primary end points were seizure-freedom rates, operating time, and complication rates.

RESULTS Seventeen children (age < 17 years) underwent a total of 19 robot-assisted SEEG electrode implantations. In total, 265 electrodes were implanted. Twelve children went on to have EZ resection: 4 demonstrated Engel class I outcomes, whereas 2 had Engel class II outcomes, and 6 had Engel class III-IV outcomes. Of the 5 patients who did not have resection, 2 underwent thermocoagulation. One child reported transient paresthesia associated with 2 small subdural hematomas, and 3 other children had minor asymptomatic intracranial hemorrhages. There were no differences in complication rates, rates of resective epilepsy surgery, or seizure freedom rates between this cohort and the preceding 18 children who underwent Talairach frame-based SEEG. The frameless robot-assisted technique was associated with shorter operating time $(p<0.05)$.

CONCLUSIONS Frameless robot-assisted SEEG is a safe and effective means of identifying the EZ in children with pharmacoresistant partial epilepsy. Robot-assisted SEEG is faster than the Talairach frame-based method, and has equivalent safety and efficacy. The former, furthermore, facilitates more electrode trajectory possibilities, which may improve the localization of epileptic networks.

https://thejns.org/doi/abs/10.3171/2018.1.PEDS17435

KEYWORDS epilepsy; intracranial recordings; pediatric neurosurgery; robotics; stereotactic neurosurgery

I $\mathrm{N}$ patients with focal pharmacoresistant epilepsy, the epileptogenic zone (EZ) is defined as that region of brain tissue that when removed results in seizure freedom. Thus, safe and accurate identification of the EZ is the crux of effective epilepsy surgery. When the EZ cannot be identified by noninvasive means (e.g., video electro- encephalography and MRI), implantation of intracranial electrodes to record directly from the brain is sometimes necessary to delineate the EZ. Stereoelectroencephalography (SEEG) is the method of planning and implanting percutaneous intracerebral electrodes based on a customized preimplantation anatomo-electro-clinical hypothesis, and

ABBREVIATIONS CAH = corticoamygdalohippocampectomy; CHUGA = Centre Hospitalier Universitaire Grenoble-Alpes; EZ = epileptogenic zone; FEF = frontal eye field; $\mathrm{IPH}=$ intraparenchymal hemorrhage; MPRAGE = magnetization-prepared rapid acquisition gradient echo; OR = operating room; ROSA = Robotic Stereotactic Assistance; SDH = subdural hematoma; SEEG = stereoelectroencephalography; VNS = vagal nerve stimulator.

SUBMITTED August 4, 2017. ACCEPTED January 24, 2018.

INCLUDE WHEN CITING Published online April 20, 2018; DOI: 10.3171/2018.1.PEDS17435. 
has been used in Europe for invasive delineation of the EZ since the 1950s. ${ }^{28}$ Numerous reports demonstrate that SEEG is a safe and effective technique for the identification of the EZ in both children and adults. ${ }^{7}$

Recent advances in neuroimaging, stereotaxy, and robotics have reinvigorated and expanded interest in SEEG as a method for localizing the EZ, and have resulted in increased interest in SEEG at epilepsy centers around the world. ${ }^{10}$ Specifically, the development of robotic devices to assist with stereotactic implantation of percutaneous intracerebral electrodes has made SEEG electrode implantation techniques more accessible at centers that do not use a Talairach or Leksell frame. Thus, numerous pediatric and adult epilepsy centers are now using robot-assisted SEEG electrode implantation techniques to localize the EZ. Given the recent development of robotic techniques, the safety, efficacy, and best practices for robot-assisted SEEG electrode implantation are not yet established, particularly in children. Additionally, there is considerable variation in SEEG electrode implantation techniques across centers, with differences in techniques seen at nearly every stage of the operation. However, the relative advantages and disadvantages of technical nuances at each stage of the procedure have yet to be determined.

The purpose of this manuscript is to describe the robot-assisted SEEG electrode implantation technique currently in use at the Centre Hospitalier Universitaire Grenoble-Alpes (CHUGA) for children. Furthermore, we also describe the frameless registration that is possible with this robot, and the importance of this technique as it relates to young children. Our program has more than 30 years of experience with SEEG, and thus has had the experience necessary to refine the SEEG technique to minimize complications and optimize localization of the EZ. We review the outcomes in our first 19 frameless robot-assisted SEEG electrode implantations (in children $<17$ years of age) to report on the safety and efficacy of pediatric SEEG electrode implantation at our center. In our experience, it appears that robot-assisted SEEG electrode implantation is as safe and efficacious for ultimate seizure freedom as our previous Talairach frame-based method.

\section{Methods}

\section{The SEEG Electrode Implantation Technique}

Patient Selection and Preoperative Management

Patients are selected for evaluation by SEEG when they suffer from focal pharmacoresistant epilepsy and noninvasive investigations fail to adequately localize the EZ. ${ }^{15}$ At CHUGA, approximately $50 \%$ of candidates for resective epilepsy surgery meet these criteria, and therefore are offered SEEG electrode implantation for localization of the EZ. Of course, patients are not candidates for SEEG if they would not ultimately be candidates for resection of an epileptic focus.

Prior to surgery, anticoagulants and antiplatelet agents are discontinued. Any antiepileptic drugs associated with bleeding abnormalities (i.e., valproate) are also discontinued and replaced with an appropriate medication. During the course of SEEG recordings (typically lasting 1-2 weeks), medications are progressively reduced as necessary to record at least one spontaneous seizure.

\section{Preimplantation Neuroimaging}

Once patients are selected for SEEG electrode implantation, they undergo a standardized preimplantation neuroimaging protocol that consists of the following elements: 1) high-resolution postcontrast MRI (magnetization-prepared rapid acquisition gradient echo [MPRAGE] sequence ${ }^{6}$; 2) thin-slice postcontrast Robotic Stereotactic Assistance (ROSA)-protocol CT; and 3) a catheterbased diagnostic angiogram formatted to a CT. Using the ROSA robot, no frame is required, and instead 5 bone fiducials are implanted under local anesthesia-either 1 day prior to SEEG electrode implantation or in the operating room (OR) at the time of SEEG implantation (typically the latter).

\section{Electrode Implantation Strategy and Planning}

The SEEG exploration strategy is discussed at a multidisciplinary epilepsy conference that includes both epileptologists and neurosurgeons experienced in SEEG. The electrode implantation strategy is selected to address an individualized anatomo-electro-clinical hypothesis, which is formulated on the basis of clinical semiology, neuropsychology, MRI findings, and long-term video EEG recordings (Micromed), with a system 10-20 and additional electrodes for the system 10-10 (FT9, FT10, TP9, and TP10), and interictal FDG-PET CT images fused with 3D T1-weighted MR images. ${ }^{18,19}$ As a principle, SEEG exploration should be tailored to evaluate the following: 1) the EZ (including seizure onset zone and early spread); 2) alternative but less likely EZ hypotheses; 3 ) the border of the EZ; 4) eloquent cortex in the region of the EZ; and 5) the relationship between the EZ and any potentially related lesions (and the epileptogenicity of such lesions). ${ }^{18}$ In the pediatric population, understanding the relationship between the lesion and any potentially extrinsic epileptic networks is crucial because in many cases the EZ extends outside the lesion. ${ }^{2}$ In our opinion, following these principles optimizes the possibility of localizing the EZ while providing necessary resection boundaries and functional mapping.

After the exploration strategy is planned, electrode trajectories are planned to execute it (Fig. 1). At our center, ROSA (Zimmer Biomet) software is used to coregister first the contrast CT to the contrast MR image, and then the angiogram to the CT scan. Electrode trajectories are then selected to 1) maximize gray matter coverage at target sites (and sulcal banks en route to targets); 2) not injure vasculature; and 3) when possible, minimize the number of electrodes used. Prior to completion, entry points are checked to ensure that none are overlapping. An important distinction between robotic SEEG and Talairach frame SEEG is that robotic SEEG does not require completely orthogonal trajectories, and thus trajectories can deviate from fully orthogonal ones to optimize coverage of gray matter.

\section{Robot Positioning, Head Fixation, and Registration}

Patients are brought to the OR and undergo general 

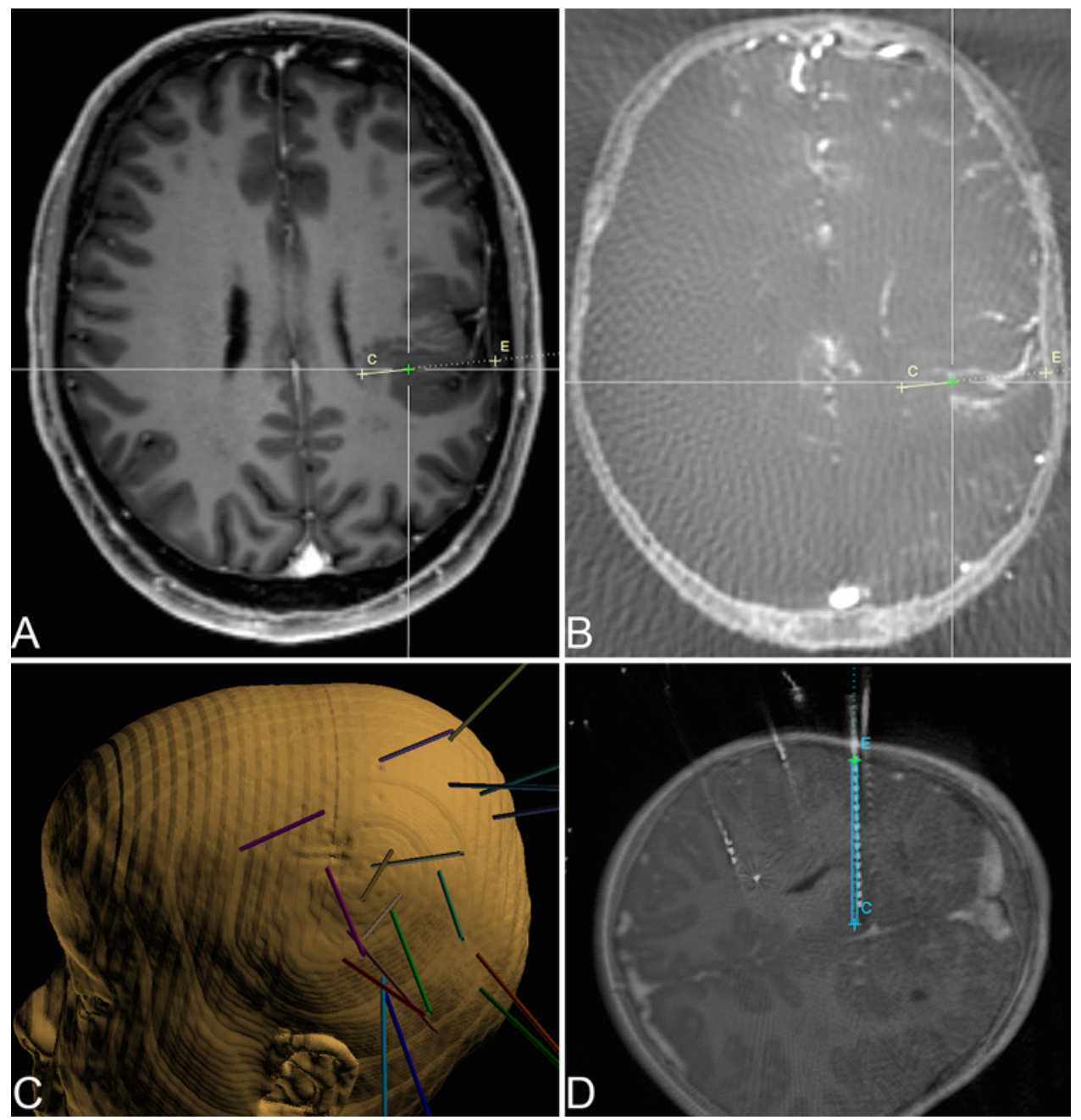

FIG. 1. Robot-assisted SEEG electrode implantation planning and intraoperative adjustment. A: Axial MPRAGE MRI view demonstrating apparently avascular SEEG electrode trajectory exploring a cortical dysplasia. B: Axial view of left carotid angiogram at the same position as panel A, demonstrating a vascular structure in the electrode trajectory. C: A 3D scalp view of a planned SEEG electrode implantation, which is used to determine the order of electrode implantation. D: Fusion of intraoperative postimplantation CT and MPRAGE MR image demonstrating position of electrode relative to planned trajectory. In this case, the electrode was advanced $4.0 \mathrm{~mm}$. Figure is available in color online only.

anesthesia. The head is positioned in a stereotactic frame that is required as a headholder, and the elbow of the robot is positioned approximately $70 \mathrm{~cm}$ from the patient's head (Fig. 2). In our opinion, fixation with a stereotactic frame is essential to ensure stereotactic accuracy of the implantation. We use a frame that is rigid, with 3 or 4 head fixation points to minimize the mobility of the head, which is mandatory when using the robotic arm and, we believe, optimizes stereotactic accuracy.

Next, the robot is registered as follows. After the scalp is prepared in the usual fashion, 5 SEEG guidance screws are inserted in the skull; these are used as fiducial markers. A 3D rendering of the entry sites from the ROSA software is examined carefully to ensure that these fiducial markers do not overlap with the eventual electrode insertion sites. We use an intraoperative CT unit (O-Arm; Medtronic) to obtain a CT scan that includes the fiducial markers, which is then coregistered to the preoperative stereotactic CT by using the ROSA software. Alternatively, if fiducials are placed the day before, a CT scan is obtained preoperatively. The ROSA is then registered using these fiducials, and we consider an error measurement $<0.8 \mathrm{~mm}$ acceptable. The guidance screws used as fiducials are maintained in position after electrode implantation in case the patient is taken back to the OR for further electrode implantation.

\section{Electrode Implantation}

The robotic implantation is initialized and the first trajectory is selected. Through the robotic guidance instrument, a power drill is used to enter the skull directly through the scalp (Fig. 3). Bone debris is irrigated from the skull opening and a sharp coagulation electrode is inserted to open the dura mater. A guidance screw $(20,25$, 30 , or $35 \mathrm{~mm}$, with the length depending on the depth of bone) is inserted into the skull. A stylet measured to the length of the target is then inserted to initiate the electrode trajectory. Care is taken to minimize the egress of CSF after opening the dura, but prior to electrode implantation, 


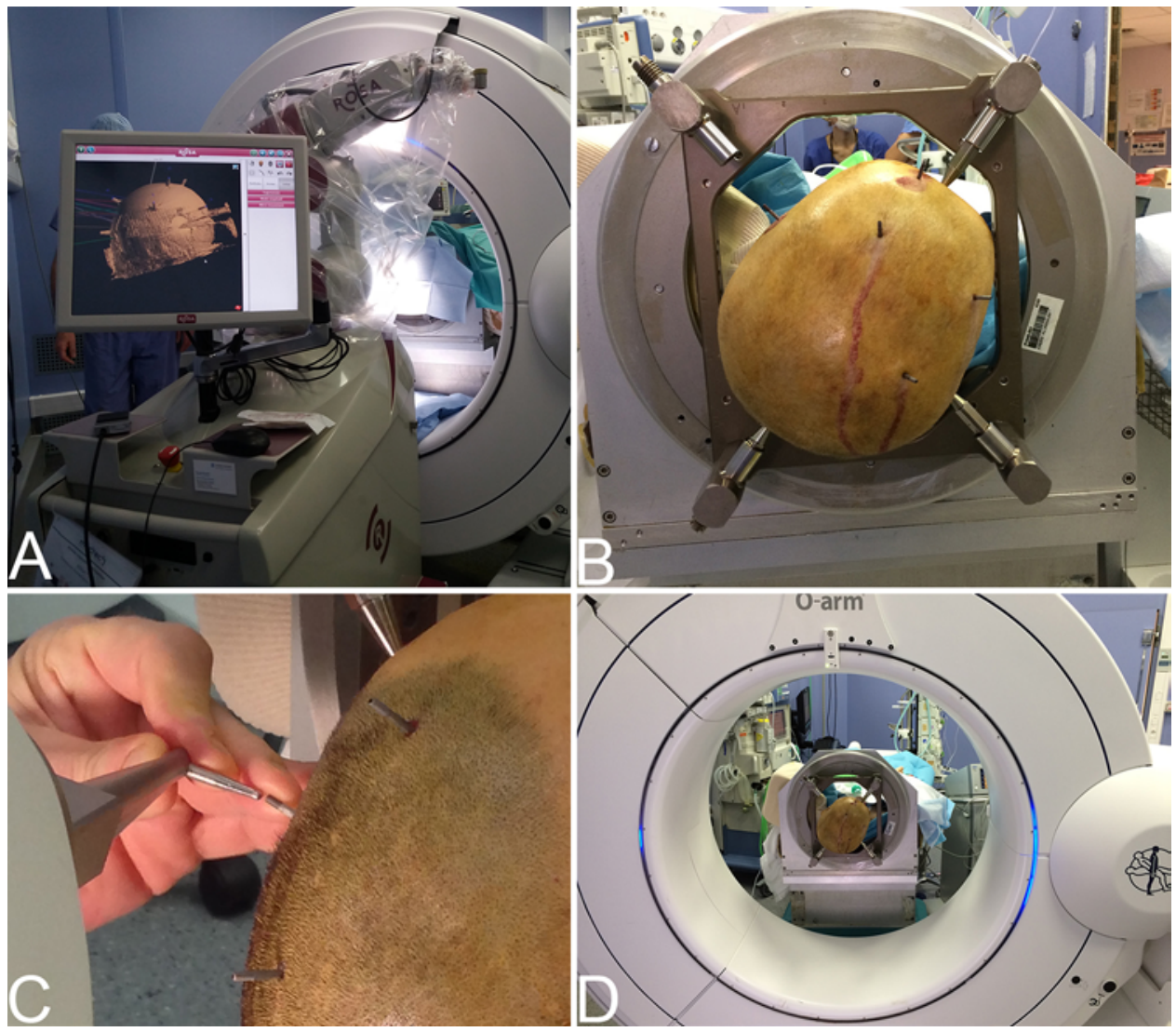

FIG. 2. Photographs of the OR setup, patient positioning, robot registration, and intraoperative CT. A: Operating room setup for frameless robot-assisted SEEG electrode implantation. B: The head is fixed in a stereotactic frame to maximize exposure of scalp entry points. Five bone fudicial markers are placed for registration. C: Registration to fudicial markers using the robotic arm. D: Use of intraoperative CT to first, obtain a CT with bone fiducial markers (if not obtained the prior day), and then to confirm the position and adjust, if necessary, implanted SEEG electrodes. Figure is available in color online only.

because this may result in slight alterations of brain position. Finally, the electrode is inserted directly through the guidance screw and is then secured to the screw at its final position.

\section{Confirmation and Correction of Electrode Positions}

Electrode positions are then confirmed using intraoperative CT. With the patient's head still fixed in the stereotactic frame, an O-arm CT scan is obtained and coregistered to the preoperative CT by using the ROSA software. Electrode positions on the postimplantation CT scan are then compared with the planned positions on preoperative MRI. Minor modifications are then made to electrode depth as necessary. If electrode positions are displaced from the planned trajectory such that they are not engaged with the gray matter site from which recording is necessary, then the guidance bolt and electrode are repositioned. After repositioning of electrodes, an additional O-arm CT scan is obtained to again confirm electrode position. A sterile dressing is then placed.

\section{Postoperative Management, Electrode Removal, and Resection Planning}

After implantation, children are admitted to a step- down unit, where they undergo monitoring for several hours prior to transfer to the pediatric floor. The SEEG recordings can begin within hours of implantation. Antiepileptic drugs are tapered as needed to promote seizures. Once the EZ is satisfactorily localized, electrodes are removed in the OR under reversible fast general anesthesia.

\section{Retrospective Review Methodology}

To evaluate the relative safety and efficacy of robotassisted SEEG, we performed a retrospective review of all recent (after 2013), pediatric (age < 17 years), robot-assisted SEEG implantation operations at our institution. As a comparison group, we retrospectively reviewed the same number of our most recent consecutive Talairach framebased SEEG implantations. For both groups, we reviewed medical records including OR reports, clinic visits, and inpatient records. We specifically looked at inpatient and clinic follow-up records for any evidence of SEEG-related complications and, when applicable, postintervention seizure freedom rates.

\section{Results}

A summary of the characteristics and results of children undergoing robot-assisted SEEG (group 1) is present- 

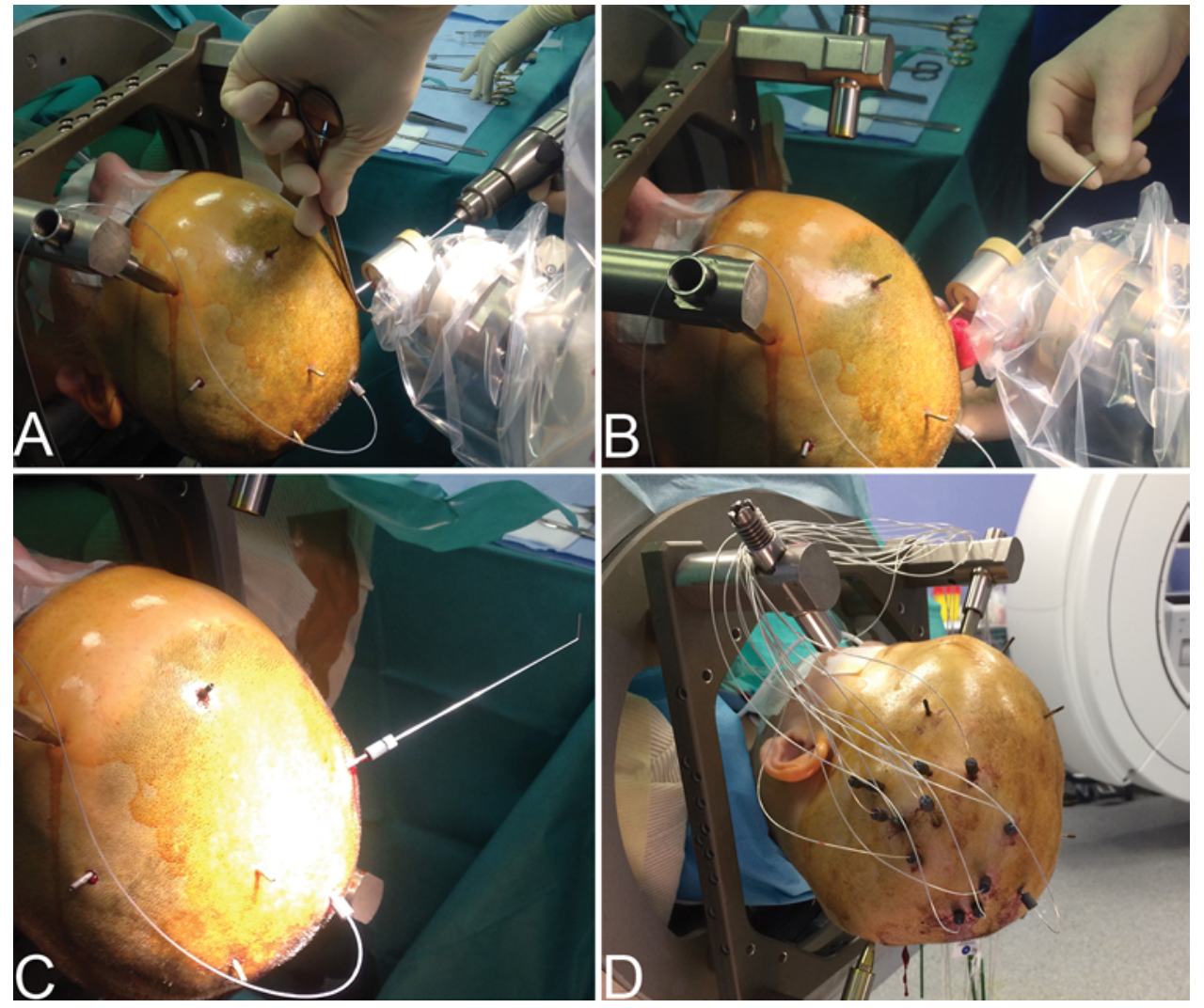

FIG. 3. Photographs of the SEEG implantation technique. This figure illustrates our technique for SEEG electrode implantation. A: After a trajectory is selected, the robotic arm navigates to the targeted entry point. A high-speed drill is placed through a port targeting the entry point provided by the robotic arm. B: Next, the dura is coagulated and opened percutaneously. C: Upon opening of the dura, a stylet is measured for the target and gently advanced to the target. The stylet is kept in place until the electrode can be inserted to avoid CSF egress. D: Electrodes are precisely measured and then "soft-passed" to the target. This panel reflects a completed implantation. Figure is available in color online only.

ed in Table 1. Seventeen children (age < 17) underwent 19 robot-assisted SEEG electrode implantations at CHUGA performed by a single surgeon (D.H.). The mean age at implantation was 11.3 years, with a range of 3-17 years. In total, 265 electrodes were implanted using the robotassisted technique for an average of 14 electrodes per implantation. Sixteen implantations were unilateral (13 left and 3 right) and 3 implantations were bilateral ( 2 of which were predominately left).

A summary of children who underwent Talairach frame-based SEEG, which is presented as a comparison group, is given in Table 2. Eighteen children (age < 17) underwent 19 Talairach frame-based SEEG electrode implantations at CHUGA by a single surgeon (D.H.). The mean age in group 2 was 11.3 years, with a range of 6-16 years. In group 2, a total of 264 electrodes were implanted for an average of 14 electrodes per implantation. Fifteen implantations were unilateral ( 8 left and 7 right) and 4 were bilateral ( 2 of which were predominately left).

The patient characteristics at the time of each SEEG electrode implantation are compared in Table 3 by using the 2-sample Student t-test for continuous variables and Fisher's exact test for categorical data, with a significance threshold set at alpha $=0.05$. Comparing group 1 and group 2, there were no significant differences in 1) the age at seizure onset; 2) age at SEEG electrode implantation; 3) sex; 4) whether there was a relevant lesion on MRI; 5) whether the epilepsy was found to be extratemporal (including temporal plus epilepsy); or 6) number of implanted electrodes.

Total OR time was compared for each group. The average OR time for group 1 was 5:04 and the average for group 2 was 5:52. This difference was statistically significant (t-test, $\mathrm{p}<0.05)$.

In group 1, 2 children underwent repeat SEEG implantations. In 1 child (Table 1, implantations 8 and 9), a right temporal, parietal, insular, and opercular SEEG exploration was initially performed, with localization of the EZ to the right temporal lobe and insula. Thus, a right corticoamygdalohippocampectomy $(\mathrm{CAH})$ and insulo-opercular corticectomy was performed with no improvement in seizures. Two years later, at age 17, the child returned and a second SEEG implantation was performed, this time for a bilateral insulo-opercular exploration. Given the finding of multifocal epilepsy from this second exploration, the child underwent vagal nerve stimulator (VNS) implantation.

The second child in group 1 to have a repeat SEEG electrode implantation (Table 1, implantations 17 and 18) initially underwent a right parietal, insular, and occipi- 
TABLE 1. Clinical data in 17 pediatric patients undergoing robot-assisted SEEG electrode implantation

\begin{tabular}{|c|c|c|c|c|c|c|c|c|c|}
\hline $\begin{array}{l}\text { Implant } \\
\text { No. }\end{array}$ & Sex & $\begin{array}{l}\text { Age at } \\
\text { Onset }\end{array}$ & $\begin{array}{l}\text { Age at } \\
\text { SEEG } \\
\text { (yrs) }\end{array}$ & MRI Findings & $\begin{array}{c}\text { SEEG } \\
\text { Hypothesis }\end{array}$ & $\begin{array}{l}\text { OR } \\
\text { SEEG } \\
\text { Time }\end{array}$ & Intervention & $\begin{array}{l}\text { Seizure } \\
\text { Outcome* }\end{array}$ & $\begin{array}{l}\text { SEEG-Related } \\
\text { Complications }\end{array}$ \\
\hline 1 & $\mathrm{~F}$ & $3 \mathrm{mos}$ & 13 & $\begin{array}{l}\text { Lt T signal abnormality, It I } \\
\text { encephalomalacia, It P } \\
\text { pachygyria }\end{array}$ & Lt F-T-I & $6: 35$ & Lt F corticectomy & IVB & None \\
\hline 2 & $\mathrm{M}$ & 6 yrs & 14 & NRL & Lt PS & $4: 15$ & $\mathrm{TC}$ & NA & None \\
\hline 3 & M & Birth & 6 & Multiple tubers (>10) & Rt T-P & $7: 53$ & Rt T-P corticectomy & IIIA & None \\
\hline 4 & $\mathrm{~F}$ & $7 \mathrm{yrs}$ & 9 & NRL & Lt F & $5: 14$ & Lt F corticectomy & IIB & $2 \mathrm{SDH}$ \\
\hline 5 & M & 4 mos & 14 & $\begin{array}{l}\text { Rt T-P junction morphologi- } \\
\text { cal abnormality }\end{array}$ & Lt F-T-P-I & $6: 15$ & Lt F-T-P corticectomy & IIIA & $\begin{array}{c}\text { Transient paresthesia, } 2 \\
\text { SDH (not correlated) }\end{array}$ \\
\hline 6 & $\mathrm{~F}$ & $5 \mathrm{yrs}$ & 6 & $\begin{array}{l}\text { Lt hemispheric encephalo- } \\
\text { malacia }\end{array}$ & Lt F-P & $5: 20$ & Lt F corticectomy & IA & None \\
\hline 7 & $\mathrm{~F}$ & 10 yrs & 12 & NRL & Rt F-I & $4: 11$ & Rt F corticectomy & IA & None \\
\hline $8 \dagger$ & $\mathrm{F}$ & $7 \mathrm{yrs}$ & 15 & NRL & Rt T-P-I-Op & 5:06 & Rt T-I-Op corticectomy & IVB & None \\
\hline $9 \dagger$ & $\mathrm{F}$ & $7 \mathrm{yrs}$ & 17 & NRL & Bilat I-Op & $5: 50$ & VNS & $\begin{array}{l}\mathrm{NA}- \\
\text { multifocal }\end{array}$ & Focal edema \\
\hline 10 & $\mathrm{M}$ & 6 yrs & 14 & NRL & Lt PS & $4: 08$ & $\mathrm{TC}$ & NA & None \\
\hline 11 & $\mathrm{~F}$ & $13 \mathrm{yrs}$ & 15 & $\begin{array}{l}\text { Lt hyperintensity FLAIR } \\
\text { mesial T-O }\end{array}$ & Lt T-O & $4: 58$ & Lt T-O corticectomy & IVB & None \\
\hline 12 & M & $1 \mathrm{yr}$ & 14 & $\begin{array}{l}\text { Lt F surgical encephaloma- } \\
\text { lacia }\end{array}$ & Lt PS & $4: 39$ & $\mathrm{Lt} \mathrm{CAH}+\mathrm{I}$ & IVB & $1 \mathrm{IPH}$ \\
\hline 13 & M & 6 yrs & 11 & $\begin{array}{l}\text { Lt abnormal F gyration of sup } \\
\quad \& \text { middle F gyri }\end{array}$ & Lt F-P & $5: 00$ & Lt F corticectomy & IA & None \\
\hline 14 & $\mathrm{~F}$ & 4 yrs & 11 & Rt amygdala hypertrophy & Rt F-T-I & $5: 30$ & $\mathrm{Rt} \mathrm{CAH}$ & $\| \mathrm{B}$ & None \\
\hline 15 & $\mathrm{M}$ & Birth & 18 & Bifrontal encephalomalacia & Lt F & $3: 26$ & Lt F corticectomy & NA & None \\
\hline 16 & M & $18 \mathrm{mos}$ & 4 & Rt F dysplasia & Lt F & $4: 29$ & Lt F corticectomy & IA & None \\
\hline $17 \ddagger$ & $\mathrm{F}$ & $8 \mathrm{yrs}$ & 10 & NRL & Rt P-I-O & $3: 31$ & NA & NA & See 2nd CT scan \\
\hline $18 \ddagger$ & $F$ & $8 \mathrm{yrs}$ & 10 & NRL & Rt F & NA & Rt F corticectomy & NA & $1 \mathrm{SDH}, 1 \mathrm{IPH}$ \\
\hline 19 & M & $5 \mathrm{mos}$ & 3 & Lt large P-T-O dysplasia & F-P-T-O-I & NA & Pending & NA & None \\
\hline
\end{tabular}

$\mathrm{C}=$ cingulate; $\mathrm{F}, \mathrm{FP}=$ frontal, frontal pole; $\mathrm{I}=$ insular; implant = implantation; $\mathrm{MTS}=$ mesial temporal sclerosis; $\mathrm{NA}=$ not applicable; $\mathrm{NRL}=$ no relevant lesion; $\mathrm{O}=\mathrm{oc}-$ cipital; $\mathrm{Op}=$ opercular; $\mathrm{P}=$ parietal; $\mathrm{PS}=$ perisylvian; sup = superior; $\mathrm{T}=$ temporal; $\mathrm{TC}=$ thermocoagulation.

* Engel classification.

† or $\ddagger$ Denotes the same patient, but different SEEG electrode implantations.

tal implantation that demonstrated electrophysiological initiation incongruent with seizure semiology. Thus, additional SEEG electrodes were placed in the frontal lobe to include the frontal eye field (FEF) during the same hospital stay. The EZ was localized to the right FEF, and a right FEF resection was performed. Seizure outcome is pending.

One child in group 2 had repeat SEEG electrode implantation (Table 2, implantations 2 and 19). Initially, he underwent a left temporal, parietal, and occipital implantation; however, the seizure onset zone was not localized at that time. A subsequent implantation 3 years later with more electrode coverage of the perirolandic cortex and the insula was consistent with the EZ being localized to arm motor cortex, and a thermocoagulation was performed.

\section{Complications Related to SEEG}

We analyzed records for evidence of any SEEG-related complications - to include infection (deep or superficial), hemorrhage, medical complications, and hardware-related complications. As part of our protocol, most patients underwent a routine CT scan after electrode removal, and these CT scans were reviewed for any radiographic evidence of complications. Complications observed in groups 1 and 2 are summarized in Tables 1 and 2, respectively. Based on postoperative CT imaging, every patient demonstrated multiple small electrode entry site hemorrhages. These were not considered to be complications unless they extended beyond the electrode entry site. In group 1,1 patient experienced transient paresthesia associated with 2 small subdural hematomas (SDHs), and 4 other patients had asymptomatic minor intracranial bleeding. In group 2, 1 patient experienced a headache postoperatively that was associated with confined electrode tract hemorrhage (<1-mm thickness), and another child had a small SDH. In neither group 1 nor group 2 did any patient suffer a complication with permanent consequences. There was no evidence of postimplantation infection in either group.

There was no difference in the rate of SDH $(\mathrm{p}=0.18)$ 
TABLE 2. Clinical data in 18 pediatric patients undergoing Talairach frame-based SEEG electrode implantation

\begin{tabular}{|c|c|c|c|c|c|c|c|c|c|}
\hline $\begin{array}{l}\text { Implant } \\
\text { No. }\end{array}$ & Sex & $\begin{array}{l}\text { Age at } \\
\text { Onset }\end{array}$ & $\begin{array}{c}\text { Age at } \\
\text { SEEG } \\
\text { (yrs) }\end{array}$ & MRI Findings & $\begin{array}{c}\text { SEEG } \\
\text { Hypothesis }\end{array}$ & $\begin{array}{c}\text { OR } \\
\text { SEEG } \\
\text { Time }\end{array}$ & Intervention & $\begin{array}{l}\text { Seizure } \\
\text { Outcome* }\end{array}$ & $\begin{array}{c}\text { SEEG } \\
\text { Complications }\end{array}$ \\
\hline 1 & M & $7 \mathrm{yrs}$ & 10 & Bilat P-O ulegyria & Lt T-P-O & $6: 30$ & P-O lesionectomy & $\mathrm{IA}$ & $\begin{array}{l}\text { HA, small It T } \\
\quad \text { IPH }\end{array}$ \\
\hline $2 \dagger$ & M & $18 \mathrm{mos}$ & 9 & NRL & Lt T-P-O & $6: 30$ & None & NA & None \\
\hline 3 & M & $18 \mathrm{mos}$ & 6 & Lt ATL dysplasia & Lt T-O-I & $6: 30$ & Lt CAH & IIB & None \\
\hline 4 & M & $3 \mathrm{yrs}$ & 10 & NRL & Rt T-I & $6: 30$ & Rt CAH & IA & None \\
\hline 5 & M & 3 days & 6 & Rt pst T-O focal megalencephaly & Rt F-P-I & $4: 35$ & Rt P corticectomy & IIIA & None \\
\hline 6 & M & $1 \mathrm{yr}$ & 11 & $\begin{array}{l}\text { Rt increased FLAIR, rt HPC; possible rt } \\
\text { pst T heterotopia }\end{array}$ & F-T-I-C & 5:05 & None & NA & None \\
\hline 7 & M & $11 \mathrm{mos}$ & 10 & Lt I transmantle sign & F-T-I & $6: 30$ & F corticectomy & IID & None \\
\hline 8 & M & $7 \mathrm{yrs}$ & 13 & $\begin{array}{l}\text { Lt T \& I atrophy; increased caudate T1- } \\
\text { weighted signal }\end{array}$ & Lt F-T-I & $5: 25$ & $\mathrm{Lt} \mathrm{CAH+I}$ & IVB & None \\
\hline 9 & M & 4 yrs & 7 & NRL & Rt F & $4: 55$ & Rt F corticectomy & $\mathrm{IA}$ & None \\
\hline 10 & M & $7 \mathrm{mos}$ & 14 & Rt parietal DNET resection & I-Op & $6: 05$ & None & NA & None \\
\hline 11 & $\mathrm{~F}$ & 17 mos & 16 & Rt HPC atrophy; rt T microcystic lesion & F-T & $4: 00$ & T corticectomy & $\mathrm{IA}$ & None \\
\hline 12 & M & $9 \mathrm{yrs}$ & 15 & Lt T-P atrophy; It HPC increased FLAIR & T-P-O & $5: 45$ & None & NA & $1 \mathrm{SDH}$ \\
\hline 13 & $M$ & $3 \mathrm{mos}$ & 9 & Lt F-T-I edema & Lt F-T-I & $7: 25$ & Lt F corticectomy & IID & None \\
\hline 14 & $\mathrm{~F}$ & $5 \mathrm{yrs}$ & 11 & $\begin{array}{l}\text { Lt precentral lesion suggestive of cortical } \\
\text { dysplasia }\end{array}$ & Lt F & $5: 58$ & Lt F corticectomy & IB & None \\
\hline 15 & $\mathrm{~F}$ & $12 \mathrm{yrs}$ & 16 & Rt F-T ischemic lesion & Rt F-T-I & $6: 10$ & Rt F-T corticectomy & IVB & None \\
\hline 16 & $\mathrm{~F}$ & $11 \mathrm{yrs}$ & 13 & NRL & Rt F & $6: 29$ & Rt F corticectomy & $\mathrm{IA}$ & None \\
\hline 17 & $M$ & $7 \mathrm{mos}$ & 12 & Lt PS \& I atrophy & Lt F-T-I & $7: 40$ & $\mathrm{TC}$ & NA & None \\
\hline 18 & $M$ & 4 yrs & 16 & NRL & Bilat $\mathrm{F}$ & $4: 10$ & VNS, DBS & NA & None \\
\hline $19 \dagger$ & M & $18 \mathrm{mos}$ & 12 & NRL & Lt T-P-O-I & $5: 25$ & TC & NA & None \\
\hline
\end{tabular}

$\mathrm{ATL}$ = anterior temporal lobe; DBS = deep brain stimulation; DNET = dysembryoplastic neuroepithelial tumor; $\mathrm{HA}=$ headache; HPC = hippocampal; pst = posterior.

* Engel classification.

$\dagger$ Denotes the same patient, with 2 separate SEEG electrode implantations.

or intraparenchymal hemorrhage (IPH) $(\mathrm{p}=1.0)$ between group 1 and group 2 as determined by a Fisher exact test.

\section{Seizure Outcomes}

Seizure outcomes were analyzed for all children who underwent resection after SEEG and had at least 1 year of clinical follow-up. In group 1, 12 children underwent resection, 2 underwent thermocoagulation, and 1 underwent placement of a VNS (after a second SEEG electrode implantation proved multifocal epilepsy). Of the 12 children who underwent resection, 4 had complete seizure freedom (Engel class I), 2 had rare disabling seizures (class II), 2 had worthwhile improvement in seizures (class III), and 4 had no worthwhile improvement (class IV).

In group 2, 12 children underwent resection. Of those 12 children, 6 had complete seizure freedom (Engel class I), 3 had rare disabling seizures (class II), 1 had worthwhile improvement in seizures (class III), and 2 had no worthwhile improvement in seizures (class IV).

When considering only children who went on to undergo resection, there was no difference in rates of seizure freedom (Engel class I) between the 2 groups $(\mathrm{p}=0.68)$.

\section{Discussion}

In this study, we demonstrated the safety and efficacy of our technique for robot-assisted SEEG electrode implantation in the pediatric population. We found similar rates of complications, rates of patients advancing to resection, and ultimate postoperative seizure freedom rates. However, based on our results, robot-assisted SEEG electrode implantation seems more efficient, requiring less time in the operating room (see Results). Notably, children in both group 1 (robot-assisted) and group 2 (Talairach framebased) had no permanent SEEG-related complications, and they had low rates of transient SEEG-related complications (1 patient in each group had symptomatic hemorrhagic complications that were transient), which highlights the low complication rate of SEEG electrode implantation

TABLE 3. Comparison of group 1 and 2 patient characteristics

\begin{tabular}{lccc}
\hline \multicolumn{1}{c}{ Characteristic } & Group 1 & Group 2 & p Value \\
\hline Age at onset (yrs) & 4.8 & 4.5 & 0.75 \\
\hline Age at SEEG (yrs) & 11.4 & 11.4 & 1 \\
\hline Sex (female) & 10 & 4 & 0.09 \\
\hline Lesional & 10 & 14 & 0.09 \\
\hline Extratemporal & 18 & 15 & 0.33 \\
\hline No. of electrodes (mean) & 14.0 & 13.9 & 0.95 \\
\hline
\end{tabular}


in children at experienced centers, regardless of whether or not a robot is used.

Although SEEG has been the standard of care at many European epilepsy centers since the $1950 \mathrm{~s}, 5,28,29$ it is now gaining popularity at epilepsy centers throughout North America. ${ }^{12-14,24,31}$ Although SEEG electrode implantation techniques have been in use in North America since the $1970 \mathrm{~s},{ }^{25}$ advances in neuroimaging and robotics have made SEEG more accessible. In fact, several pediatric epilepsy centers in the US have invested in robotic devices to perform robot-assisted SEEG electrode implantation. However, the optimal technique for SEEG electrode implantation and its safety remain topics of intense debate. ${ }^{12,22}$ The technique we describe here is the result of more than 30 years of experience with SEEG electrode implantation at our center.

The relative safety and most appropriate application of different intracranial recording techniques is a current topic of debate..$^{16}$ Recently, several groups have examined the risks associated with intracranial recordings to localize the EZ using either SEEG ${ }^{8,22}$ or craniotomy with grid strip recordings. ${ }^{3,4,17,26,27}$ In 2013, Cardinale et al. ${ }^{8}$ reviewed complication rates in their series of 500 SEEG electrode implantations. In this large single-center series (from an experienced center), SEEG electrode implantation-related complications were rare. In this series, 13 intracranial hemorrhages were reported (7 of which were reportedly "minor"), with 4 of these hemorrhages requiring surgical intervention, and 3 resulting in permanent neurological deficits. Additionally, in this series, a 3-year-old child developed severe hyponatremia after SEEG electrode implantation and died from massive cerebral edema.

Recently, Mullin et al..$^{22}$ performed a meta-analysis of the SEEG literature to assess complication rates of SEEG. In this meta-analysis, the results of 30 SEEG studies (describing 2624 SEEG electrode implantations) were analyzed, reporting a $1.0 \%$ prevalence of hemorrhagic complications $(0.7 \% \mathrm{IPH}, 0.4 \% \mathrm{SDH}$, and $0.3 \%$ epidural hematoma); $0.8 \%$ prevalence of infectious complications $(0.9 \%$ intracranial abscess, $1.4 \%$ superficial skin infections, and $0.6 \%$ meningitis); and $0.6 \%$ prevalence of permanent neurological deficits. Interestingly, of the studies examined by Mullin et al., there is a wide range of complication ratesreflecting either heterogeneity of complication rates at different centers or differences in the reporting of complication rates. The low complication rates of SEEG reported by Cardinale et al. ${ }^{8}$ and Mullin et al..$^{22}$ are consistent with the complication rates we present here, which probably reflects our relatively small sample size and the low rate of complications related to SEEG electrode implantation. Additionally, our complication rate is consistent with those of other purely pediatric SEEG series. ${ }^{30}$

In the SEEG literature, there are inconsistencies regarding the need for angiographic imaging to plan SEEG electrode implantations. Some groups attest that double contrast MRI is sufficient to ensure safety, whereas others have reported that catheter-based angiography is necessary. ${ }^{1,9,14,23,25}$ As described in our technique presented here, it is our view that catheter-based angiography is important for safely planning SEEG electrode implantations. Additionally, catheter-based angiography may decrease the risk of cerebral infarction during SEEG-guided thermocoagulation procedures. Because the rate of hemorrhagic complications associated with SEEG is low regardless of whether a catheter-based angiogram is used, exactly how much the hemorrhage rate is decreased by angiographic imaging remains unknown. However, Mirzayan et al. ${ }^{21}$ recently reported that 27 of 146 SEEG electrode trajectories were modified based on cerebral C-arm CT angiography, which supports a role for angiographic imaging in safe SEEG electrode implantation.

It is our view that our head fixation and robot registration preferences contribute to the safety and accuracy of SEEG electrode implantation. In our experience, a stereotactic head fixation device (e.g., Leksell frame or similar device) rather than a standard craniotomy head fixation (e.g., Mayfield clamp) should be used, given that stereotactic head fixation devices have been the gold standard for stereotactic procedures requiring a high degree of accuracy (e.g., deep brain stimulation). Although the Leksell head fixation device is our preference, some SEEG groups have published their technique in which the Mayfield head clamp is used,,$^{20}$ and there is some evidence that frameless stereotaxy performed using the Mayfield head clamp may be as accurate as frame-based stereotaxy techniques for certain applications. ${ }^{11}$ However, a recent manuscript reported up to $10 \%$ of electrodes deviating more than $3 \mathrm{~mm}$ when using a Mayfield head clamp. ${ }^{20}$

Furthermore, it is our preference to place 5 bone fiducial markers spaced evenly across the head, which we believe is best for achieving accuracy of both superficial and deep SEEG targets. In contrast, it is possible that face-tracing methods may limit the accuracy of targets remote from the face, although there are no currently available data to support this claim. Maintaining placement of bone fudicials during the monitoring period is also helpful because the fiducials can be used for additional stereotactic imaging should a repeated implantation be necessary based on the initial findings of SEEG recording.

In this paper, we have emphasized the importance of SEEG surgical technique for the safety and efficiency of SEEG electrode implantations. However, it is important to note that the success of a SEEG electrode implantation for localizing the EZ hinges on the development of a strong anatomo-electro-clinical hypothesis prior to implantation. ${ }^{15,18}$ Despite safe and accurate implantation of SEEG electrodes, if targets are not appropriately selected during the implantation planning phase, the implantation will not accurately localize the EZ. Therefore, working with a team of neurologists and neurosurgeons experienced with the SEEG methodology is essential to building a successful SEEG program.

Unfortunately, given the sample size and heterogeneity of groups 1 and 2, it is not possible for us to make strong conclusions about the relative efficacy of our frameless robot-assisted and Talairach frame-based SEEG electrode implantation techniques with regard to localization of the EZ. Additionally, although robot-assisted SEEG electrode implantation appears to be safe in the cohort of patients we present here, given the low rate of SEEG-related complications we cannot make conclusive statements about the safety of robot-assisted SEEG electrode implantation 
compared to other traditional methods. Furthermore, our rates of complications, proceeding from SEEG to resection, and ultimate seizure freedom are probably influenced by many years of experience at our center, and it is not known how these methods will influence epilepsy surgery at less experienced SEEG centers.

Another limitation of our study is that the cases described here represent our earliest cases with the ROSA robot in children, and thus the operating times may become even shorter as our group gains experience. Other SEEG programs have shown shorter OR times; $;{ }^{20}$ however, the exact reasons for this are not known.

\section{Conclusions}

Robot-assisted SEEG is as safe and effective as Talairach frame-based SEEG electrode implantation, but requires less OR time and can be performed in a frameless fashion. The emergence of robotic technology has allowed for widespread proliferation of SEEG electrode implantation techniques to centers outside of Europe. Furthermore, the robot enables planning and implantation of oblique SEEG trajectories that can maximize the coverage of gray matter sites. Further work with larger numbers of patients is necessary to determine whether or not robot-assisted SEEG electrode implantation influences ultimate postresection seizure freedom rates.

\section{References}

1. Afif A, Chabardes S, Minotti L, Kahane P, Hoffmann D: Safety and usefulness of insular depth electrodes implanted via an oblique approach in patients with epilepsy. Neurosurgery 62 (5 Suppl 2):ONS471-ONS480, 2008

2. Aghakhani Y, Kinay D, Gotman J, Soualmi L, Andermann F, Olivier A, et al: The role of periventricular nodular heterotopia in epileptogenesis. Brain 128:641-651, 2005

3. Albert GW, Dahdaleh NS, Reddy C, Hansen DR, Vogel TW, Kawasaki $\mathrm{H}$, et al: Postoperative radiographic findings in patients undergoing intracranial electrode monitoring for medically refractory epilepsy. J Neurosurg 112:449-454, 2010

4. Arya R, Mangano FT, Horn PS, Holland KD, Rose DF, Glauser TA: Adverse events related to extraoperative invasive EEG monitoring with subdural grid electrodes: a systematic review and meta-analysis. Epilepsia 54:828-839, 2013

5. Bancaud J, Angelergues R, Bernouilli C, Bonis A, BordasFerrer M, Bresson M, et al: [Functional stereotaxic exploration (stereo-electroencephalography) in epilepsies.] Rev Neurol (Paris) 120:448, $1969(\mathrm{Fr})$

6. Brant-Zawadzki M, Gillan GD, Nitz WR: MP RAGE: a three-dimensional, T1-weighted, gradient-echo sequenceinitial experience in the brain. Radiology 182:769-775, 1992

7. Cardinale F, Casaceli G, Raneri F, Miller J, Lo Russo G: Implantation of stereoelectroencephalography electrodes: a systematic review. J Clin Neurophysiol 33:490-502, 2016

8. Cardinale F, Cossu M, Castana L, Casaceli G, Schiariti MP, Miserocchi A, et al: Stereoelectroencephalography: surgical methodology, safety, and stereotactic application accuracy in 500 procedures. Neurosurgery 72:353-366, 2013

9. Cardinale F, Pero G, Quilici L, Piano M, Colombo P, Moscato A, et al: Cerebral angiography for multimodal surgical planning in epilepsy surgery: description of a new threedimensional technique and literature review. World Neurosurg 84:358-367, 2015

10. Chabardes S, Abel TJ, Cardinale F, Kahane P: Commentary: Understanding stereoelectroencephalography: what's next? Neurosurgery 82:E15-E16, 2018
11. Dorward NL, Alberti O, Palmer JD, Kitchen ND, Thomas DG: Accuracy of true frameless stereotaxy: in vivo measurement and laboratory phantom studies. Technical note. J Neurosurg 90:160-168, 1999

12. Gonzalez-Martinez J, Bulacio J, Alexopoulos A, Jehi L, Bingaman W, Najm I: Stereoelectroencephalography in the "difficult to localize" refractory focal epilepsy: early experience from a North American epilepsy center. Epilepsia 54:323-330, 2013

13. Gonzalez-Martinez J, Mullin J, Bulacio J, Gupta A, Enatsu R, Najm I, et al: Stereoelectroencephalography in children and adolescents with difficult-to-localize refractory focal epilepsy. Neurosurgery 75:258-268, 2014

14. Gonzalez-Martinez J, Mullin J, Vadera S, Bulacio J, Hughes $\mathrm{G}$, Jones S, et al: Stereotactic placement of depth electrodes in medically intractable epilepsy. J Neurosurg 120:639-644, 2014

15. Hoffmann D, Russo GL, Cossu M: Stereoelectroencephalography, in Lüders HO (ed): Textbook of Epilepsy Surgery. Boca Raton: CRC Press, 2008, pp 945-959

16. Jayakar P, Gotman J, Harvey AS, Palmini A, Tassi L, Schomer D, et al: Diagnostic utility of invasive EEG for epilepsy surgery: Indications, modalities, and techniques. Epilepsia 57:1735-1747, 2016

17. Johnston JM Jr, Mangano FT, Ojemann JG, Park TS, Trevathan E, Smyth MD: Complications of invasive subdural electrode monitoring at St. Louis Children's Hospital, 19942005. J Neurosurg 105 (5 Suppl):343-347, 2006

18. Kahane P, Francione S: Stereoelectroencephalography, in Lüders HO (ed): Textbook of Epilepsy Surgery. Boca Raton: CRC Press, 2008, pp 649-658

19. Lamarche F, Job AS, Deman P, Bhattacharjee M, Hoffmann D, Gallazzini-Crépin C, et al: Correlation of FDG-PET hypometabolism and SEEG epileptogenicity mapping in patients with drug-resistant focal epilepsy. Epilepsia 57:2045-2055, 2016

20. Miller BA, Salehi A, Limbrick DD Jr, Smyth MD: Applications of a robotic stereotactic arm for pediatric epilepsy and neurooncology surgery. J Neurosurg Pediatr 20:364-370, 2017

21. Mirzayan MJ, von Roden M, Bulacio J, von Podewils F, Gonzalez-Martinez J: The usefulness of intraoperative cerebral $\mathrm{C}$-arm CT angiogram for implantation of intracranial depth electrodes in stereotactic electroencephalography procedure. Stereotact Funct Neurosurg 94:10-17, 2016

22. Mullin JP, Shriver M, Alomar S, Najm I, Bulacio J, Chauvel $\mathrm{P}$, et al: Is SEEG safe? A systematic review and meta-analysis of stereo-electroencephalography-related complications. Epilepsia 57:386-401, 2016

23. Mullin JP, Smithason S, Gonzalez-Martinez J: Stereo-electro-encephalo-graphy (SEEG) with robotic assistance in the presurgical evaluation of medical refractory epilepsy: a technical note. J Vis $\operatorname{Exp}(\mathbf{1 1 2}):$ e53206, 2016

24. Munyon C, Sweet J, Luders H, Lhatoo S, Miller J: The 3-dimensional grid: a novel approach to stereoelectroencephalography. Neurosurgery 11 (Suppl 2):127-134, 2015

25. Olivier A, Boling WW, Tanriverdi T: Techniques in Epilepsy Surgery: The MNI Approach. New York: Cambridge University Press, 2012

26. Rolston JD, Englot DJ, Cornes S, Chang EF: Major and minor complications in extraoperative electrocorticography: a review of a national database. Epilepsy Res 122:26-29, 2016

27. Rolston JD, Ouyang D, Englot DJ, Wang DD, Chang EF: National trends and complication rates for invasive extraoperative electrocorticography in the USA. J Clin Neurosci 22:823-827, 2015

28. Schijns OE, Hoogland G, Kubben PL, Koehler PJ: The start and development of epilepsy surgery in Europe: a historical review. Neurosurg Rev 38:447-461, 2015 
29. Talairach J, Bancaud J: Lesion, "irritative" zone and epileptogenic focus. Confin Neurol 27:91-94, 1966

30. Taussig D, Chipaux M, Lebas A, Fohlen M, Bulteau C, Ternier J, et al: Stereo-electroencephalography (SEEG) in 65 children: an effective and safe diagnostic method for pre-surgical diagnosis, independent of age. Epileptic Disord 16:280-295, 2014

31. Wu S, Kunhi Veedu HP, Lhatoo SD, Koubeissi MZ, Miller JP, Lüders HO: Role of ictal baseline shifts and ictal highfrequency oscillations in stereo-electroencephalography analysis of mesial temporal lobe seizures. Epilepsia 55:690-698, 2014

\section{Disclosures}

Dr. Amorim-Leite is a consultant for Abbot, Novartis, UCB, and Cyberonics. Dr. Chabardes is a consultant for Zimmer Biomet.

\section{Author Contributions}

Conception and design: Abel, Varela Osorio, Amorim-Leite, Chabardes. Acquisition of data: Abel, Varela Osorio, AmorimLeite, Chabardes. Analysis and interpretation of data: Abel, Amorim-Leite, Minotti, Chabardes. Drafting the article: Abel, Amorim-Leite, Chabardes. Critically revising the article: Abel, Amorim-Leite, Mathieu, Minotti, Chabardes. Reviewed submitted version of manuscript: Abel, Kahane, Minotti, Hoffmann, Chabardes. Approved the final version of the manuscript on behalf of all authors: Abel. Statistical analysis: Mathieu. Administrative/technical/material support: Kahane, Minotti, Hoffmann, Chabardes. Study supervision: Kahane, Chabardes.

\section{Correspondence}

Taylor J. Abel: The Hospital for Sick Children, Toronto, ON, Canada.taylor.abel@sickkids.ca. 